\title{
Gene Expression Profile of RNA N1-methyladenosine methyltransferases
}

\author{
Mingzhu Rao ${ }^{1}$ \\ ${ }^{1}$ School of Pharmaceutical Science and Technology, Tianjin University, Tianjin China
}

\begin{abstract}
N1-methyladenosine $\left(\mathrm{m}^{1} \mathrm{~A}\right)$ is a kind of common and abundant methylation modification in eukaryotic mRNA and long-chain non-coding RNA. Nucleoside methyltransferase (MTase) of $\mathrm{m}^{1} \mathrm{~A}$ is a diverse protein family, which is characterized by the presence of methyltransferases like domains and conserved S-adenosylmethionine (SAM) binding domains formed by the central sevenstranded beta-sheet structure. However, comprehensive analysis of the gene expression profile of such enzymes has not been performed to classify them according to evolutionary criteria and to guide the functional prediction. Here, we conducted extensive searches of databases to collect all members of previously identified $\mathrm{m}^{1} \mathrm{~A}$ RNA methyltransferases. And we report bioinformatics studies on gene expression profile based on evolutionary analysis, sequence alignment, expression in tissues and cells within the family of RNA methyltransferases. Our analysis showed that the base modification behavior mediated by $\mathrm{m}^{1} \mathrm{~A}$ RNA methyltransferases evolved from invertebrate, and the active sites of $\mathrm{m}^{1} \mathrm{~A}$ RNA methyltransferases were highly conserved during the evolution from invertebrates to human. And $\mathrm{m}^{1} \mathrm{~A}$ RNA methyltransferases have low tissue and cell specificity.
\end{abstract}

\section{Introduction}

RNA is a key link between DNA and protein in the process of genetic information transmission, but the level of synthesized protein is not necessarily positively correlated with the level of mRNA, suggesting the importance of post-transcriptional modification of RNA ${ }^{1}$. To date, more than 100 different types of base modification behavior have been identified ${ }^{2}$. Internal modification occurs in many types of RNA, of which N6-methyladenosine $\left(\mathrm{m}^{6} \mathrm{~A}\right)$, the most common modification in eukaryotes, refers to methylation of adenylate at the sixth nitrogen position ${ }^{3}$. $\mathrm{m}^{1} \mathrm{~A}$ modification is different from $\mathrm{m}^{6} \mathrm{~A}$ in that it occurs on the first $\mathrm{N}$ atom of the adenine base group and has a positive charge under physiological conditions. $\mathrm{m}^{1} \mathrm{~A}$ was first found in non-coding RNA such as rRNA and tRNA and widely existed in prokaryotic and eukaryotic mRNA ${ }^{4}$. Compared with $\mathrm{m}^{6} \mathrm{~A}$, the content of $\mathrm{m}^{1} \mathrm{~A}$ in human and mouse tissues is very limited, and the content of $\mathrm{m}^{1} \mathrm{~A}$ in mRNA is less than one-tenth of $\mathrm{m}^{6} \mathrm{~A}$, but it can greatly affect protein-RNA interaction and RNA secondary structure through electrostatic effect. In addition, $\mathrm{m}^{1} \mathrm{~A}$ modification is related to translation initiation sites, and transcripts containing $\mathrm{m}^{1} \mathrm{~A}$ have higher translation efficiency ${ }^{5} \cdot \mathrm{m}^{1} \mathrm{~A}$ is a positively charged nucleotide modification, and the methyl group on $\mathrm{m}^{1} \mathrm{~A}$ will block the Watson-Crick base complementary pairing of adenine, which also makes them have an important contribution to the formation of tRNA structure ${ }^{6}$. In tRNA $\mathrm{m}^{1} \mathrm{~A}$ is mainly present at position 58 of the tRNA, and it is also found in small amounts at positions $9,14,22$, and 57 of the tRNA.
Based on the latest single-base resolution m1 A sequencing technology, the researchers found that $\mathrm{m}^{1} \mathrm{~A}$ modification is mainly concentrated in mRNA cap and 5'UTR region, and $\mathrm{m}^{1} \mathrm{~A}$ modification also exists in mitochondrialencoded transcripts ${ }^{7}$. Because $\mathrm{m}^{1} \mathrm{~A}$ contains a methyl group at the N1 position that can interfere with WatsonCrick base pairs, $\mathrm{m}^{1} \mathrm{~A}$ can cause reverse transcription to stop and can also lead to erroneous synthesis. Based on this, Dominissini ${ }^{5}$ and $\mathrm{Li}^{4}$ developed transcriptome-wide sequencing methods to identify and map $\mathrm{m}^{1} \mathrm{~A}$ in mRNA. These studies indicate that $\mathrm{m}^{1} \mathrm{~A}$ is reversible and is mainly concentrated near the start codon of eukaryotic mRNA. However, the binding proteins of $\mathrm{m}^{1} \mathrm{~A}$ are still unclear, and its specific functions and mechanisms need to be further explored. Further identification of $\mathrm{m}^{1} \mathrm{~A}$ binding proteins will help to understand the functional role of $\mathrm{m}^{1} \mathrm{~A}$. The discovery of $\mathrm{m}^{1} \mathrm{~A}$ is a new RNA marker positively correlated with translation, adding an exciting new dimension to the regulation of post-transcriptional gene expression mediated by RNA modification ${ }^{5}$.

There are a variety of RNA methyltransferases and demethylases in cells, as well as various methyl binding proteins. Under their combined action, different types of RNA undergo dynamic changes of methylation and demethylation to regulate various physiological processes. The number of studies about human RNA methyltransferases has been increasing rapidly in the past few years, and both structural characteristics and biological functions are the research hotspots. However, there are only few studies on the expression profile of RNA methyltransferases ${ }^{8,9}$. Our project focuses on all $\mathrm{m}^{1} \mathrm{~A}$ RNA methyltransferases. Based on the gene 
expression level and sequence alignment of the enzymes obtained from some databases, the gene expression profile of $\mathrm{m}^{1} \mathrm{~A}$ methyltransferase was studied by means of bioinformatics. To understand the origin of these enzymes in detail, we summarized the species without $\mathrm{m}^{1} \mathrm{~A}$ methyltransferase orthologues together with the corresponding discussion of the gene sequences and structure to verify the evolution of RNA methylation modifications. Moreover, expression and localization of $\mathrm{m}^{1} \mathrm{~A}$ RNA methyltransferases across tissues and cells better indicate the specificity of gene expression. The clustering analysis of m1A RNA methyltransferases expression profiles reveals their tissue and cell specificities. The results of the present studies provide a further understanding of the evolutionary profile of RNA modification and valuable insights into their functions.

\section{Methods}

\section{1 $\mathrm{m}^{1}$ A RNA Methyltransferases Searches}

Human enzymes with RNA $\mathrm{m}^{1} \mathrm{~A}$ methylation function were collected from the PubMed database of the National Center for Biotechnology Information (https://pubmed.ncbi.nlm.nih.gov/). All the results were used as queries to carry out the second round of searching, and the same procedure was followed for the retrieval of key residues of the RNA methyltransferases. The information on RNA methyltransferases' active residues, substrates, and binding sites with ligands was collected from previously published sources.

\subsection{Analysis of Species Tree}

The species tree including 259 species from Ensembl database

(http://asia.ensembl.org/info/about/speciestree.html,

Ensembl Release 100) $)^{10}$ was downloaded for further analysis. Ensembl is a genome browser for vertebrate genomes. The species tree from Ensembl describes the evolutionary relationship of primates, rodents, laurasiatheria, afrotheria, xenarthral, birds, reptiles, amphibians, fish, cyclostomes, two chordates, and three other eukaryotes. And the species of $\mathrm{m}^{1} \mathrm{~A}$ RNA methyltransferases that don't have any orthologue with the enzyme's gene are labeled in the evolutionary tree. The species tree was displayed and edited by a tool called Interactive Tree of Life (iTOL) ${ }^{11}$.

\subsection{Structure Analysis}

Crystal structures were prepared using PyMOL (The PyMOL Molecular Graphics System, Version 1.4.1, Schrodinger, LLC). Structure of $\mathrm{m}^{1} \mathrm{~A}$ RNA methyltransferase in complex with SAM has been deposited in the Protein Data Bank with PDB IDs: TRMT6-TRMT61A (5CCB).

\subsection{Sequence Alignment}

Twenty representative species were selected to do sequence alignment based on various stages of vertebrate evolution including Saccharomyces cerevisiae, Drosophila melanogaster, Caenorhabditis elegans, Ciona intestinalis, Ciona savignyi, Lamprey (Petromyzon marinus), Hagfish (Eptatretus burgeri), Elephant shark (Callorhinchus milii), Coelacanth (Latimeria chalumnae), Zebrafish (Danio rerio), Tropical clawed frog (Xenopus tropicalis), Tuatara (Sphenodon punctatus), Mainland tiger snake (Notechis scutatus), Painted turtle (Chrysemys picta bellii), Australian saltwater crocodile (Crocodylus porosus), Platypus (Ornithorhynchus anatinus), Mouse (Mus musculus), Great Tit (Parus major), Sooty mangabey (Cercocebus atys), and Human (Homo sapiens). The protein sequences of the different $\mathrm{m}^{1} \mathrm{~A}$ RNA methyltransferases from the above mentioned 20 species were downloaded from the Ensembl database. Multiple sequence alignments of the methyltransferases were generated using MEGA and displayed the results using ClustalX. Creates a multiple alignment of all database hits for each query sequence.

\subsection{Analysis of Gene Expression in Tissues and Cells}

To analyze the RNA expression of $\mathrm{m}^{1} \mathrm{~A}$ RNA methyltransferases in different human cells and tissues, we used the RNA data from the Human Protein Atlas (HPA) database (Version: 19) ${ }^{12}$, which contains six atlas parts, among which the Tissue Atlas shows the expression of proteins across major tissues in the human body and the Cell Atlas shows expression and localization of proteins in single cells. Normalized eXpression (NX) values in major tissues and cell lines were used to perform the analysis, which was created by combining the data from the three transcriptomics datasets (HPA, GTEx, and FANTOM5). Heatmap visualization was implemented with HemI (Heatmap Illustrator, version 1.0) ${ }^{13}$ to compare the expression level of $\mathrm{m}^{1} \mathrm{~A}$ RNA methyltransferases in tissues and cells. HemI clustering method is the default average linkage, and the similarity metric is calculated by the default Pearson distance.

\section{Results and Discussion}

\subsection{The Properties of m1A RNA Methyltransferases}

We found that only four enzymes were identified with $\mathrm{m}^{1} \mathrm{~A}$ methylation activity for human RNA through information collection (Table 1). These enzymes, including TRMT6, TRMT61A, TRMT61B, and TRMT10C. TRMT61A and TRMT6 are responsible for N1-methyladenine at position $58\left(\mathrm{~m}^{1} \mathrm{~A} 58\right)$ in cytoplasmic tRNAs ${ }^{14}$. TRMT6 together with the TRMT61A catalytic subunit mediates methylation of adenosine residues at the $\mathrm{N} 1$ position of a small subset of mRNAs, and N1 methylation takes place in tRNA T-loop-like structures of mRNAs and is only present at low stoichiometries ${ }^{15}$. 
TRMT61B is a kind of methyltransferase that catalyzes the formation of N1-methyladenine at position $58\left(\mathrm{~m}^{1} \mathrm{~A} 58\right)$ in various tRNAs in the mitochondrion, including tRNA(Leu) (deciphering codons UUA or UUG), tRNA(Lys) and tRNA(Ser) (deciphering codons UCA, UCU, UCG or UCC) ${ }^{16}$. And TRMT61B catalyzes the formation of 1-methyladenosine at position 947 of mitochondrial 16S rRNA and this modification is most likely important for mitoribosomal structure and function $^{17}$. In addition to tRNA N1-methyltransferase activity, it also acts as an mRNA N1-methyltransferase by mediating methylation of adenosine residues at the N1 position of MT-ND5 mRNA, leading to interfere with mitochondrial translation ${ }^{7}$. TRMT10C is also mitochondrial tRNA N1-methyltransferase involved in mitochondrial tRNA maturation ${ }^{18}$. Both TRMT61B and TRMT10C are mitochondrial RNA methyltransferases.

Table1. Number of $\mathrm{m}^{1} \mathrm{~A}$ RNA methyltransferases and RNAs based on data retrieval

\begin{tabular}{lll}
\hline RNA methyl- & Subcellular & Target RNA \\
\hline TRMT6 & Nucleus, Cell & mRNA, tRNA \\
TRMT61A & Nucleus & mRNA, tRNA \\
TRMT61B & Mitochondria & mt-tRNA, mt- \\
TRMT10C & Mitochondria, & mt-tRNA \\
\hline
\end{tabular}

mitochondrial RNA - mt-RNA

\subsection{Origin and Evolution of m1A RNA Methyltransferases}

To investigate the evolutionary history of RNA demethylases and methyltransferases genes, we performed species evolution analysis on representative species. The evolution of vertebrates has gone through the evolutionary process of chordates, cyclostomes, fish (cartilaginous fish and bony fish), amphibians, reptiles, mammals, birds, and primates ${ }^{19}$. ENSEMBL is not only a eukaryotic genome annotation project that focuses on vertebrate genomic data but also includes other organisms such as yeast, nematodes, and so on. The database contains three invertebrate eukaryotes, two invertebrate chordates, two cyclostomes, and around two hundred other vertebrates. In this study, we selected all the species in ENSEMBL mentioned above to construct the species trees (Figure 1). Some species don't have any orthologues with some RNA methyltransferases. The species without orthologues of $\mathrm{m}^{1} \mathrm{~A}$ RNA methyltransferases are marked with colored graphics in Figure 1. The evolutionary tree indicates that TRMT6 and TRMT61A appeared from Saccharomyces cerevisiae. And mitochondrial methyltransferases TRMT61B and TRMT10C appeared from Saccharomyces cerevisiae and Ecdysozoa, respectively. The extensive presence of TRMT6 and TRMT61A in early invertebrates and vertebrates suggests that the appearance of $\mathrm{m}^{1} \mathrm{~A}$ is early in RNA. Mitochondrial $\mathrm{m}^{1} \mathrm{~A}$ methyltransferases TRMT61B and TRMT10C evolved from invertebrates.

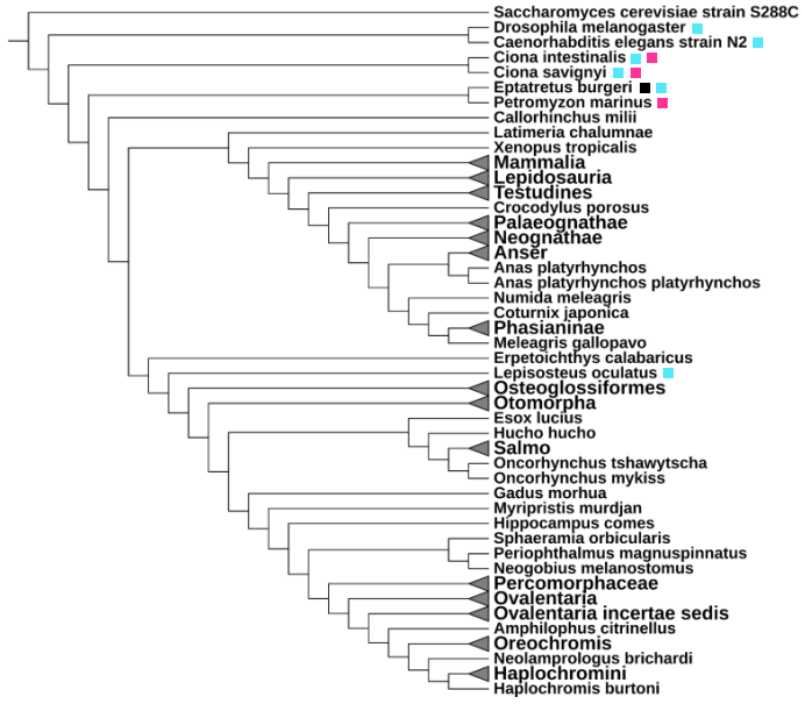

Fig1. Species trees of $\mathrm{m}^{1} \mathrm{~A}$ RNA methyltransferases. The trees were constructed based on vertebrate evolution. Species without orthologues of RNA methyltransferases of $\mathrm{m}^{1} \mathrm{~A}$ are marked with colored squares in the figure ( $\mathbf{\square}$ :without orthologues of TRMT6, : : without orthologues of TRMT61B, : without orthologues of TRMT10C).

\subsection{Verification of Active Site}

Twenty representative species are sorted in the sequence alignment according to the evolutionary process. In eukaryotes, the $\mathrm{m}^{1} \mathrm{~A} 58$ MTase located in the cytosol is composed of a catalytic protein unit from the TRM61 subfamily (TRMT61A) and an RNA-binding protein unit from the TRM6 subfamily (TRMT6). The eukaryotic complex of TRMT6-TRMT61A has been reported as a heterotetramer ${ }^{20}$. Here we display the structure of human $\mathrm{m}^{1} \mathrm{~A} 58$ MTase in complex with its cofactor and a cognate substrate, human $\mathrm{tRNA}_{3}{ }^{\text {Lys }}$ (Figure 2A). The structures provide clues to the range of tRNA conformations recognized by other tRNA-modifying enzymes to access their targets. SAM-binding sites and RNA binding sites have been previously reported ${ }^{21}$. The only hydrogenbonding side chains close to the target-binding site is Asp181, Asp181 is best positioned to interact with the A58 base. Asp181 may facilitate methyl transfer by increasing the nucleophilicity of A58 N1 and also contributes to cofactor binding ${ }^{22}$. The stereo plot of the active site of TRMT61A is shown in Figure 2B. SAMbinding MTases are characterized by several conserved sequence motifs, four of which line the SAM-binding site, and the cofactor-binding site is highly conserved in TRMT61A (Figure 2C). The TRMT61A subunit could be distinguished from TRMT6 by density for SAM in the cofactor site. In TRMT61A, SAM makes equivalent hydrogen bonds with residues from motifs. TRMT6 has an 83-residue insert in its N-terminal domain and a 98residue insert in the middle of the $\mathrm{C}$-terminal domain, both of which contribute to tRNA binding. The cofactorbinding pocket is occluded by inserted residue Arg377, which hydrogen bonds to the tRNA backbone ${ }^{21}$. TRMT6 lacks the conserved sequence motifs that line the SAMbinding pocket in SAM-dependent MTases (Figure 2D). The conserved Asp181 in TRMT61A, which has been 
implicated in catalysis, is an Ala in TRMT6. Thus, TRMT6 cannot have methylase activity and only has a tRNA-binding role. TRMT6 in Sphenodon punctatus

A

lacks RNA binding sites, indicating that the species does not have $\mathrm{m}^{1} \mathrm{~A}$ catalytic activity.
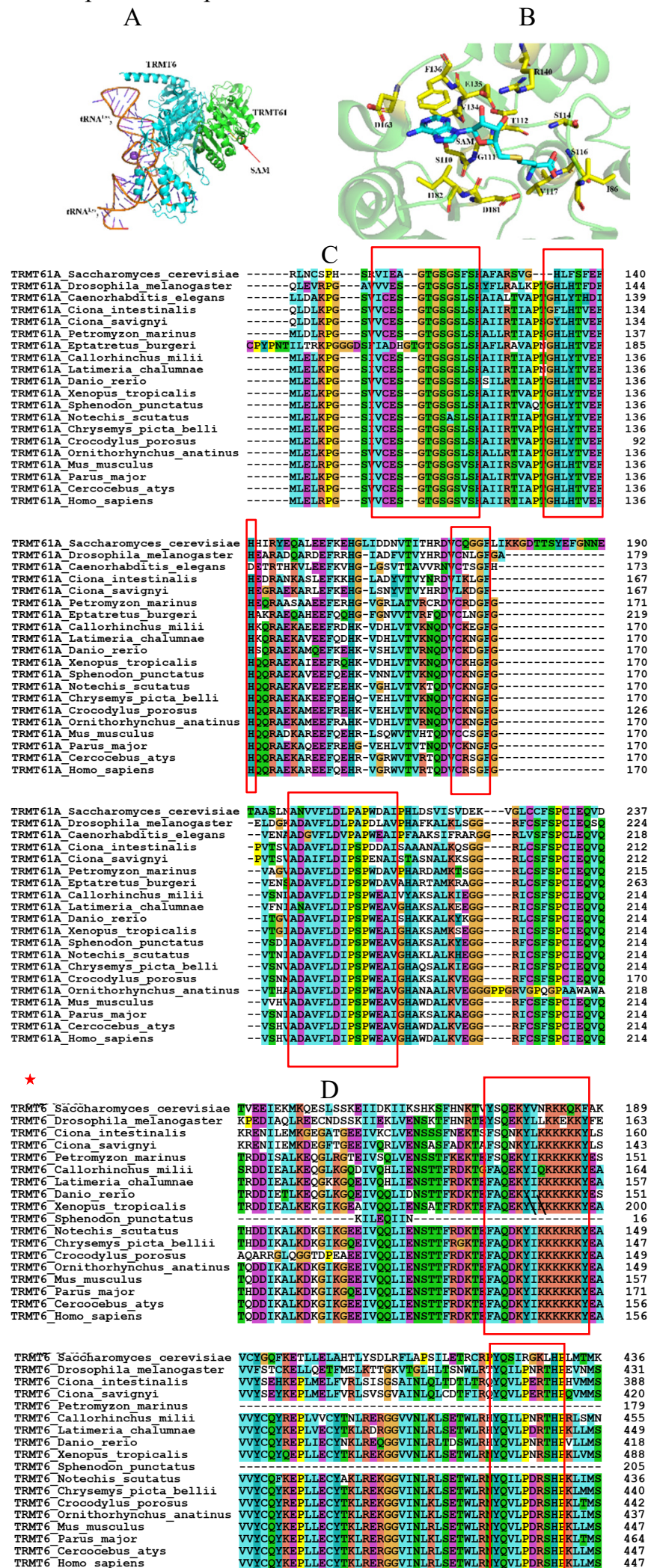

$\mathrm{D}$
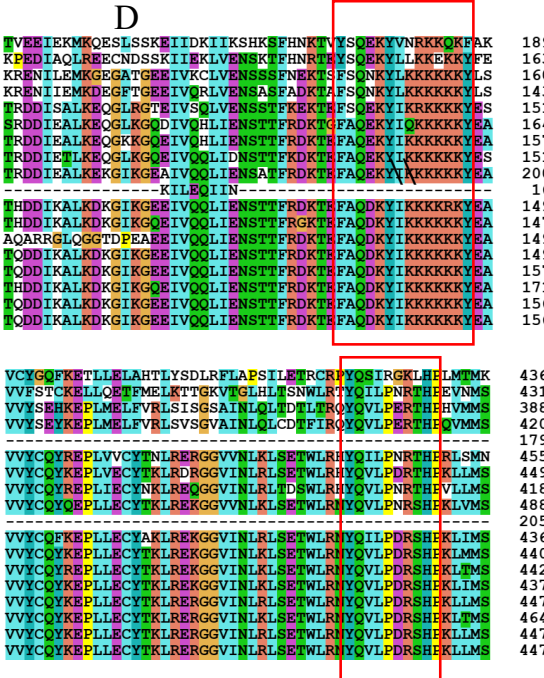

Fig2. Conserved structures of the $\mathrm{m}^{1} \mathrm{~A}$ methyltransferases. A: TRMT6-TRMT61A complex bound to tRNA ${ }^{\text {Lys }}{ }_{3}$ (PDB 5CCB); B: Binding site for SAM in the TRMT61A subunit; C: Sequence alignment of $\mathrm{m}^{1} \mathrm{~A}$ methyltransferase TRMT61A. The red box surrounding the aligned sequences identify conserved sequence motifs that surround the cofactor-binding site in SAMdependent methyltransferases. The red star identifies the Asp that is important for catalysis; D: Sequence alignment of $\mathrm{m}^{1} \mathrm{~A}$ methyltransferase TRMT6. The red box surrounding the aligned sequences indicates the RNA-binding motif. 
TRMT61B and TRMT10C are all mitochondrial tRNA methyltransferases. The identity of the enzyme catalyzing mitochondrial mRNA N1-methyltransferase is unclear. According to a report, mitochondrial mRNA N1methyltransferase activity is catalyzed by TRMT61B. According to a second report, it is mediated by TRMT10C. As both reports only tested one protein (either TRMT61B or TRMT10C), it is possible that both proteins have this activity $^{7}$. Vertebrate TRMT61B proteins were predicted to localize to the mitochondria. TRMT61B catalyzed the formation of $\mathrm{m}^{1} \mathrm{~A}$ at position 58 of mitochondrial tRNA in a SAM-dependent manner ${ }^{17}$. Sequence alignment of TRMT61B from different species shows that they possess several conserved motifs specific to SAM-dependent methyltransferases (Figure 3A), including the GxGxG sequence for SAM binding, and the conserved carboxylate motif for recognition of ribose hydroxyls. Each TRMT10C is also bound by a SAM molecule. The SAM pocket is enclosed by four extended loops highly conserved in sequence, harboring motifs I (aa 288-294), II (aa 307-323), III (aa 335-338), and IV (aa 350-360) ${ }^{18}$. Sequence alignment of TRMT10C shows that the four motifs corresponding to loops enclosing the SAM ligandbinding pocket are highly conservative in different species (Figure 3B).

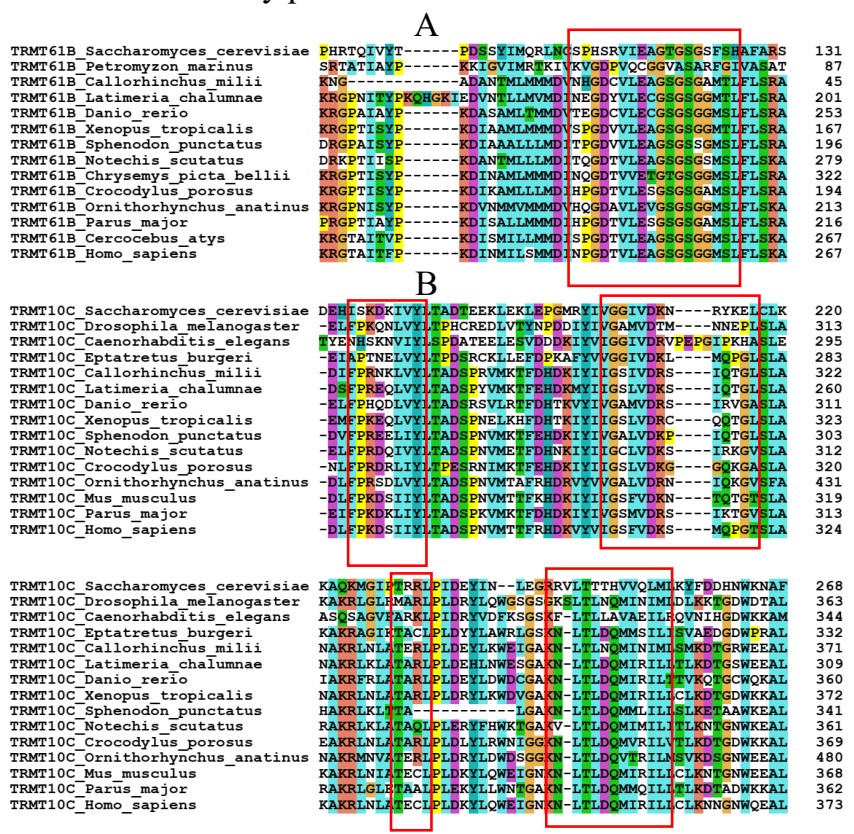

Fig3. Sequence alignment of TRMT61B and TRMT10C from different species. TRMT61B and TRMT10C are shown in A and B, respectively. Red boxes surrounding the aligned sequences indicate the SAM-binding motifs.

\subsection{RNA Expression Analysis in Tissues and Cells}

The expression of RNA methyltransferases of $\mathrm{m}^{1} \mathrm{~A}$ in normal tissues and cells was reviewed through data retrieval in the Human Protein Atlas. Clustering of transcript data in tissues and cells are shown in Figure 4A and $4 \mathrm{~B}$, respectively. In the Human Protein Atlas the specificity category must meet the requirement that expression level in a particular tissue/cell type is at least four times than any other tissue/cell type. It can be seen from Figure 4 that m1A RNA methyltransferases have low tissue specificity and cell specificity. Even though TRMT10C is highly expressed in Daudi cell lines, it still fails to meet the value requirements of cell specificity. Daudi is a kind of human lymphoma cell, but it is not clear whether the expression of TRMT10C is related to lymphoma.

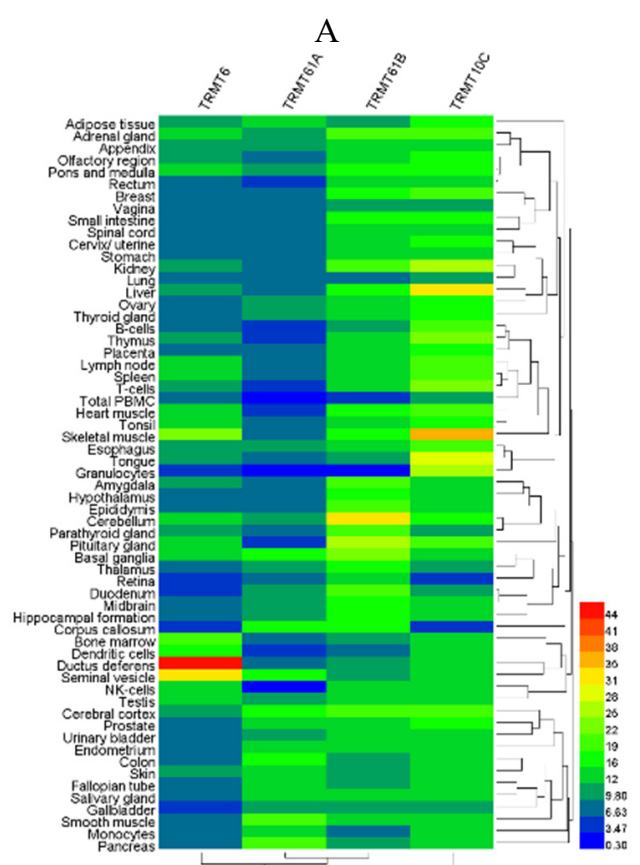




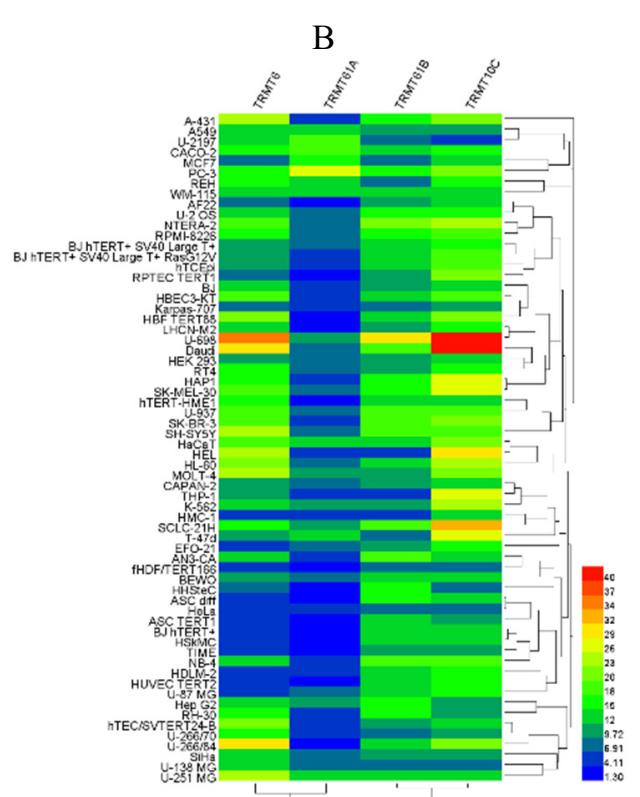

Fig4. Heatmap of RNA expression of $\mathrm{m}^{1} \mathrm{~A}$ methyltransferases in RNA. A: Heatmap in tissues; B: Heatmap in cells. The $A$ and $B$ use RNA expression level from Human Protein Atlas.

\section{Conclusion}

We summarize the types of $\mathrm{m}^{1} \mathrm{~A}$ RNA methyltransferases, all of which belong to TRMT family. Moreover, we showed the gene expression profile of the RNA methyltransferases based on species evolution and analysis of gene sequence. The extensive presence of TRMT6 and TRMT61A in early invertebrates and vertebrates suggests that the modification of $\mathrm{m}^{1} \mathrm{~A}$ in RNA may have evolved from the invertebrate period. Active sites of $\mathrm{m}^{1} \mathrm{~A}$ RNA methyltransferases are highly conserved, it is suggested that the $\mathrm{m}^{1} \mathrm{~A}$ modification in RNA is highly conserved in different evolutionary stages. In addition, the expression of RNA methyltransferases of $\mathrm{m}^{1} \mathrm{~A}$ in normal tissues and cells showed low specificity. In summary, our study improves the understanding about the gene expression profile of $\mathrm{m}^{1} \mathrm{~A}$ RNA methyltransferases and provides valuable information for future studies. Looking for the relationship between the expression level of enzymes involved in RNA modifications and tissue has broad and far-reaching significance. It will effectively promote the transformation of RNA methylation modification from basic research to drug development, and promote the treatment of clinical diseases.

\section{ACKNOWLEDGMENTS}

Financial support from National Natural Science Foundation of China (NSFC 21772143), the Natural Science Foundation of Tianjin (17JCYBJC42200, 18JCYBJC94000), Tianjin Youth 1000-Plan Talent Program and Startup Funding of Tianjin University is gratefully acknowledged.

\section{REFERENCES}

1. Yuri Motorin and Mark Helm (2011). RNA nucleotide methylation. WIREs RNA, 2, 611-631.

2. Magdalena A. Machnicka, Kaja Milanowska, Okan Osman Oglou, Elzbieta Purta, Malgorzata Kurkowska, Anna Olchowik, Witold Januszewski, Sebastian Kalinowski, Stanislaw Dunin-Horkawicz, Kristian M. Rother, Mark Helm, Janusz M. Bujnicki and Henri Grosjean (2013). MODOMICS: a database of RNA modification pathways - 2013 update. Nucleic Acids Research, 41(1), 262-267.

3. Chuan He (2010). Grand Challenge Commentary: RNA epigenetics. Nature Chemical Biology, 6, 863865.

4. Xiaoyu Li, Xushen Xiong, Kun Wang, Lixia Wang, Xiaoting Shu, Shiqing Ma and Chengqi Yi (2016). Transcriptome-wide mapping reveals reversible and dynamic N1-methyladenosine methylome, Nature Chemical Biology, 12(5), 311-316.

5. Dan Dominissini, Sigrid Nachtergaele, Sharon Moshitch-Moshkovitz, Eyal Peer, Nitzan Kol, Moshe Shay Ben-Haim, Qing Dai, Ayelet Di Segni, Mali Salmon-Divon, Wesley C. Clark, Guanqun Zheng, Tao Pan, Oz Solomon, Eran Eyal, Vera Hershkovitz, Dali Han, Louis C. Doré, Ninette Amariglio, Gideon Rechavi and Chuan He (2016). The dynamic N1methyladenosine methylome in eukaryotic messenger RNA, Nature, 530(7591), 441-446.

6. Mathias Sprinzl, Carsten Horn, Melissa Brown, Anatoli Ioudovitch and Sergey Steinberg (1998). Compilation of tRNA sequences and sequences of tRNA genes. Nucleic Acids Research, 21(13), 30113015.

7. Xiaoyu Li, Xushen Xiong, Meiling Zhang, Kun Wang, Ying Chen, Jun Zhou, Yuanhui Mao, Jia Lv, Danyang Yi, Chu Wang, Chengqi Yi and Shubing Qian (2017). Base-resolution mapping reveals distinct $\mathrm{m}^{1} \mathrm{~A}$ methylome in nuclear- and mitochondrial-encoded transcripts. Molecular cell, 68(5), 993-1005.

8. Luis Sanchez-Pulido and Miguel A Andrade-Navarro (2007). The FTO (fat mass and obesity associated) gene codes for a novel member of the non-heme dioxygenase superfamily. BMC Biochemistry, 8, 2329.

9. Marianne Pedersen Westbye, Emadoldin Feyzi, Per Arne Aas, Cathrine Broberg Vågbø, Vivi Anita Talstad, Bodil Kavli, Lars Hagen, Ottar Sundheim, Mansour Akbari, Nina-Beate Liabakk, Geir Slupphaug, Marit Otterlei and Hans Einar Krokan (2008). Human AlkB homolog 1 is a mitochondrial protein that demethylates 3-methylcytosine in DNA and RNA. Journal of Biological Chemistry, 283, 25046-25056.

10. Javier Herrero, Matthieu Muffato, Kathryn Beal, Stephen Fitzgerald, Leo Gordon, Miguel Pignatelli, Albert J Vilella, Stephen M J Searle, Ridwan Amode, Simon Brent, William Spooner, Eugene Kulesha, Andrew Yates, Paul Flicek (2016). Ensembl 
comparative genomics resources. Database: the journal of biological databases and curation, 2016, 117.

11. Ivica Letunic and Peer Bork (2016). Interactive tree of life (iTOL) v3: an online tool for the display and annotation of phylogenetic and other trees. Nucleic acids research, 44(1), 242-245.

12. Mathias Uhlen, Per Oksvold, Linn Fagerberg, Emma Lundberg, Kalle Jonasson, Mattias Forsberg, Martin Zwahlen, Caroline Kampf, Kenneth Wester, Sophia Hober, Henrik Wernerus, Lisa Björling and Fredrik Ponten (2010). Towards a knowledge-based Human Protein Atlas. Nature Biotechnology, 28(12), 12481250.

13. Wankun Deng, Yongbo Wang, Zexian Liu, Han Cheng and Yu Xue (2014). HemI: A toolkit for illustrating heatmaps. PLoS One, 9(11), 111988111993.

14. Sarah Ozanick, Annette Krecic, Joshua Andersland and James T Anderson (2005). The bipartite structure of the tRNA $\mathrm{m}^{1} \mathrm{~A} 58$ methyltransferase from $\mathrm{S}$. cerevisiae is conserved in humans. RNA, 11(8), 12811290.

15. Modi Safra, Aldema Sas-Chen, Ronit Nir, Roni Winkler, Aharon Nachshon, Dan Bar-Yaacov, Matthias Erlacher, Walter Rossmanith, Noam SternGinossar and Schraga Schwartz (2017). The $\mathrm{m}^{1} \mathrm{~A}$ landscape on cytosolic and mitochondrial mRNA at single-base resolution. Nature, 551(7679), 251-255.

16. Takeshi Chujo and Tsutomu Suzuki (2012). Trmt61B is a methyltransferase responsible for 1methyladenosine at position 58 of human mitochondrial tRNAs. RNA, 18(12), 2269-2276.

17. Dan Bar-Yaacov, Idan Frumkin, Yuka Yashiro, Takeshi Chujo, Yuma Ishigami, Yonatan Chemla, Amit Blumberg, Orr Schlesinger, Philipp Bieri, Basil Greber, Nenad Ban, Raz Zarivach, Lital Alfonta, Yitzhak Pilpel, Tsutomu Suzuki and Dan Mishmar (2016). Mitochondrial 16S rRNA is methylated by tRNA methyltransferase TRMT61B in all vertebrates. PLoS Biology, 14(9), 1002557-1002578.

18. Lien K Brzezniak, Monika Bijata, Roman J Szczesny and Piotr P Stepien (2011). Involvement of human ELAC2 gene product in $3^{\prime}$ end processing of mitochondrial tRNAs. RNA Biology, 8(4), 616-626.

19. Sudhir Kumar and S. Blair Hedges (1998). A molecular timescale for vertebrate evolution. Nature, 392(6679), 917-920.

20. Louis Droogmans, Martine Roovers, Janusz M. Bujnicki, Catherine Tricot, Thomas Hartsch, Victor Stalon and Henri Grosjean (2003). Cloning and characterization of tRNA ( $\left.\mathrm{m}^{1} \mathrm{~A} 58\right)$ methyltransferase (TrmI) from Thermus thermophilus HB27, a protein required for cell growth at extreme temperatures. Nucleic Acids Research, 31(8), 2148-2156.

21. Janet Finer-Moore, Nadine Czudnochowski, Joseph D O'Connell 3rd, Amy Liya Wang and Robert M Stroud (2015). Crystal structure of the human tRNA m(1)A58 methyltransferase-tRNA(3)(Lys) complex:
Refolding of substrate tRNA allows access to the methylation target. Journal of Molecular Biology, 427(24), 3862-3876.

22. Pierre Barraud, Béatrice Golinelli-Pimpaneau, Cédric Atmanene, Sarah Sanglier, Alain Van Dorsselaer, Louis Droogmans, Frédéric Dardel and Carine Tisné (2008). Crystal structure of Thermus thermophilus tRNA $\mathrm{m}^{1} \mathrm{~A} 58$ methyltransferase and biophysical characterization of its interaction with tRNA. Journal of Molecular Biology, 377 (2), 535-550. 